\title{
Mindset für Zeit- und \\ Handlungsspielraum: \\ Handlungsempfehlungen für \\ Führungskräfte virtueller Teams
}

\author{
Rebekka Mander, Frank Müller und Ulrike Hellert
}

\subsection{Einleitung}

Neueste Technologien beeinflussen Team- und Projektarbeit und erfordern ein Umdenken bei der Arbeitsgestaltung. Die Entwicklung von Technologien ist der Arbeitsweise vieler Teams voraus, da die Anpassung der Arbeitsprozesse lediglich schrittweise vonstattengeht (Bennett 2010). Durch eine moderne Informations- und Kommunikationstechnologie ist es möglich, dass Teammitglieder nicht gleichzeitig am selben Ort sein müssen, um gemeinsam an einer Aufgabe zu arbeiten (Chudoba et al. 2005). Die daraus entstehende virtuelle Teamarbeit zeigt sich, wenn Teams über Zeit oder Ort verteilt sind. Neben standortübergreifender Arbeit stellen auch versetzte Arbeitszeiten eine Form virtueller Teamarbeit dar. Verteilte Teams benötigen dabei neue Kooperationsansätze, um handlungsfähig zu bleiben (Lilian 2014). So gibt es beispielsweise Situationen, in denen Präsenzteams kurze Fragen durch einen einfachen Zuruf klären können. Erfolgt die Kommunikation hingegen über digitale Medien, besteht hier die erste Entscheidung in der Wahl eines angemessenen Mediums: wenn Betroffene sofort eine Antwort benötigen, ist ein Anruf meist zweckmäßiger als eine E-Mail. Anschließend muss entschieden werden, ob das Anliegen wirklich eine Störung in Form eines Anrufs rechtfertigt. Die Hürde der sofortigen Kontaktaufnahme liegt dabei höher

R. Mander $(\bowtie) \cdot$ F. Müller $\cdot$ U. Hellert

FOM Hochschule für Oekonomie \& Management gemeinnützige Gesellschaft mbH, iap Institut für Arbeit \& Personal, Essen, Deutschland

E-Mail: rebekka.mander@fom.de

F. Müller

E-Mail: frank.mueller@fom.de

U. Hellert

E-Mail: ulrike.hellert@fom.de 
als bei Präsenzarbeit. Hier könnte ein Anliegen z. B. durch Blickkontakt signalisiert werden. Diese und ähnliche Situationen erfordern ein Umdenken in der Organisation von Teamarbeit (Ale Ebrahim et al. 2009).

Führung auf Distanz wird mitunter durch die Medienwahl beeinflusst (Avolio et al. 2000). Ein Informationsaustausch zwischen Teammitgliedern findet bei virtueller Arbeit tendenziell seltener statt als bei Präsenz. Bei virtueller Teamarbeit muss eine Kontaktaufnahme proaktiv geschehen, während Begegnungen in Präsenz meist automatisch stattfinden. Zudem ist es schwieriger, Nuancen im Ausdruck aufgrund von Gestik und Mimik wahrzunehmen. In diesem Kontext zeigt sich, dass ein modernes Führungs- und Kommunikationsverständnis mit neuen Ansätzen notwendig wird. Bei Führung auf Distanz ist ein grundlegendes $\mathrm{Maß}$ an Vertrauen notwendig, da z. B. Anwesenheitskontrolle kaum noch möglich ist (Germain und McGuire 2014). Zielorientierung kann eine Orientierung an Leistungskontrolle und Bewertungen ablösen. Dies zeigt sich z. B. in einer Ablehnung von Mikromanagement als Ausdruck einer offenen Grundeinstellung (Cleary et al. 2015). So sind die Teammitglieder auch bei geringeren Möglichkeiten zum Austausch handlungsfähig. Diese Arbeitsweise erfordert jedoch einen gesteigerten Zeit- und Handlungsspielraum und eine entsprechende Einstellung bzw. ein passendes Mindset (Hofert 2018). Der Beitrag beschäftigt sich mit einem solchen Mindset.

\subsection{Theorie}

Für Menschen ist das Bedürfnis nach Kontrolle und Orientierung elementar. Das Kontrollbedürfnis zählt hierbei zu den psychischen Grundbedürfnissen, die für psychische Gesundheit und psychosoziales Wohlbefinden wichtig sind. Ferner sind es die Bedürfnisse nach Lust, Bindung und Selbstwerterhöhung, die bei allen Menschen vorhanden sind und befriedigt werden möchten (Grawe 2004, S. 185). Der Kontrollaspekt lässt sich anhand der zielorientierten Handlung verdeutlichen, wobei das Erreichen oder Nicht-Erreichen eines angestrebten Zieles die individuelle Kontrollerfahrung beeinflusst. Wird ein gesetztes Ziel erreicht, ergibt sich eine positive Kontrollerfahrung. Wird das Ziel jedoch nicht erreicht, ergibt sich entsprechend eine negative Kontrollerfahrung. Hieraus folgen entsprechende individuelle sowie arbeitsbezogene Kontrollmöglichkeiten im Hinblick auf potenzielle Zielsetzungen. Menschen mit positiven Kontrollerfahrungen werden eher bereit sein, sich zu engagieren, um ein Arbeitsziel zu erreichen.

Das Kontrollbedürfnis hat dabei Auswirkungen auf den wahrgenommenen Handlungsspielraum. Menschen, die Kontrolle anstreben, versuchen dies mit einem entsprechenden Handlungsspielraum zu verbinden, um ausreichend Ressourcen für erforderliche Flexibilität zu haben. Nach der Inkongruenztheorie von Grawe (2004) geht die Nichtbefriedigung des Kontrollbedürfnisses stets mit der Verletzung des Kontrollbedürfnisses einher, wobei Inkongruenz entsteht. Somit ist das Kontrollbedürfnis bei der Herbeiführung und Aufrechterhaltung von Arbeitszielen von besonderer Bedeutung. 
Kontrolle zu haben setzt voraus, einen gewissen Überblick über die Abläufe zu haben und zu wissen, welche Vorgänge als nächstes erfolgen. Dies verschafft Orientierung und ermöglicht wiederum, die eigenen Ziele zu realisieren. Im Job-Demand-Control-Modell (JDC) von Karasek (1979) wird der Kontrollaspekt dargestellt. Nach diesem Modell beeinflusst Kontrolle bzw. Handlungsspielraum bei der Arbeit die subjektive Belastung. Hohes Belastungsniveau bei geringer Kontrolle führt zu hoher Arbeitsanforderung. Handlungsspielraum ist somit eine wichtige Ressource, um anstehende Herausforderungen zu bewältigen (Hellert et al. 2013).

Handlungsspielräume können bereits für sich genommen in der Arbeit einen entscheidenden Einfluss auf die erlebte Beanspruchung durch Arbeitsanforderungen haben. So können Arbeitsressourcen (Handlungsspielraum, Zeitkompetenz) als Puffer belastungsreduzierend wirken und die Zielerreichung fördern (Bakker und Demerouti 2014). Handlungsspielräume sind wiederum eine wichtige Voraussetzung, um individuelle Verhaltensweisen, wie die Zeitkompetenz, zu fördern (Tegtmeier und Hellert 2015). Es sollte jedoch beachtet werden, dass Handlungsspielräume allein nicht ausreichen, um vor Überlast insbesondere in Hinblick auf Arbeitszeitnutzung zu schützen (Hellert et al. 2009). Mechanismen der individuellen Selbstkontrolle können einen guten Schutz vor Arbeitsbelastung bieten (Hollmann et al. 2005), allerdings ist auch hier zu beachten, dass Selbstkontrollmechanismen bei intensiver Nutzung Ressourcen verbrauchen (Schmidt und Neubach 2009).

Die virtuelle Arbeitswelt erfordert zunehmend agile Fähigkeiten, die zielführend, innovativ und human sind. Das Mindset, als Denk- und Handlungslogik sowohl auf individueller als auch unternehmerischer Ebene spielt hierbei eine wichtige Rolle. Hofert (2018) erläutert das Mindset im Sinne der Systemtheorie zum Zwecke des Selbsterhalts. Das äußere Umfeld prägt das individuelle und organisationale Mindset. Die digitale Transformation mit den unterschiedlichen Implikationen auf die Menschen und die Arbeitswelt wird somit auch das Mindset verändern bzw. anpassen (Hofert 2018). Agil handeln bedeutet in diesem Kontext jedoch nicht „schneller - höher - weiter“, sondern bezieht sich auf die innere Haltung zu den sich ändernden Arbeitsverhältnissen und Arbeitsmerkmalen. Hierzu zählt vor allem die Bereitschaft zum Perspektivenwechsel und zur kontinuierlichen Kompetenzentwicklung.

Die moderne Arbeitswelt ist u. a. durch Digitalisierung gekennzeichnet. Die Zusammenarbeit erfolgt dank neuester Technologien virtuell und ermöglicht zeitliche, örtliche und organisatorische Flexibilität. Führungskräfte sind zunehmend gefordert, ihr Mindset auf neue virtuelle Arbeitsprozesse umzustellen und gleichzeitig (klassisch) zu motivieren und einen wertschätzenden empathischen Umgang zu pflegen.

Zur erfolgreichen Bewältigung all der neuen Anforderungen in der digitalen Arbeitswelt steht ein solides Mittel zur Verfügung: Vertrauen. Vertrauen ist nach Luhmann (2009) ein Mechanismus, der Komplexität reduzieren kann. Das ist besonders in einer Welt nötig, die durch unbestimmt bleibende Komplexität geprägt ist. Vertrauen kann dabei Handlungsmöglichkeiten wie kooperatives Handeln generieren, die ohne Vertrauen undenkbar wären. Trotz aller Trends und Zukunftsszenarien lässt sich Handeln 
nicht völlig durch sichere Voraussicht planen. Es wird weiterhin Unsicherheiten geben, die durch zukunftsorientierte, proaktiv handelnde Führungskräfte kompensiert werden müssen. Der Erfolg stellt sich jedoch erst nach einer aktiven Handlung ein, unter Umständen aber auch dann nicht (Luhmann 2009). Da eine vollständige Kontrolle im dynamischen Umfeld nicht möglich ist, bleibt nur das Vertrauen - Vertrauen in bewährte Abläufe, in kompetente Personen oder in das organisationale Mindset. Hat ein Unternehmen positive Erfahrungen im Umgang mit Veränderungen, wird es leichter auf die eigenen Kompetenzen zu vertrauen. In der Folge werden Kommunikationsmuster optimiert, Kooperationen entstehen und Kontrolle kann als Überprüfungselement reduziert werden. Eine Führungskultur ohne Vertrauen hingegen erschwert Kooperation. Kontrollinstanzen führen zu hohem Zeitaufwand und Angst verdrängt Kreativität.

Jede noch so vertrauensvolle Führung benötigt ein System der Rückmeldung über den Projektverlauf oder den aktuellen Arbeitsprozess. Hierzu werden entsprechende Maßnahmen implementiert, die beispielsweise über regelmäßiges Prozessfeedback informieren und mögliche Handlungen induzieren. Hierzu zählen insbesondere bei virtueller Führung regelmäßige, terminierte Telefon- und/oder Videokonferenzen. Ergänzend können kommunikative Maßnahmen wie ein regelmäßiges informelles Gespräch (E-Coffee) oder ein vertrauliches Gespräch (E-Talk) bei einer Unklarheit unterstützen.

Die Führungskompetenzen beinhalten in großem Maße Verlässlichkeit für vereinbarte Regelungen. Seien es Terminabsprachen oder Aufgabeninhalte; denn Verlässlichkeit schafft Vertrauen. Gerade die Zeitkompetenz ist hier ein wichtiges und hilfreiches Element. Grundsätzlich umfasst der Kompetenzbegriff intraindividuell die Fähigkeit selbstorganisiert und kreativ auch unter Unsicherheit zielorientiert $\mathrm{zu}$ handeln. Zeitkompetenz als Ressource bei der Arbeit umfasst individuelle und organisationale Faktoren. Individuell zeigt sich Zeitkompetenz in Bezug auf die eigene Zeitwahrnehmung, beispielsweise in der Einschätzung des Pausen- und Schlafbedarfes. Organisational zeigt sie sich z. B. in Form empathischer Beurteilung der unterschiedlichen Eigenzeiten des Teams. (vgl. Hellert 2018).

Ein Mindset für virtuelle Teamarbeit beinhaltet sowohl grundlegende Faktoren wie das erforderliche Vertrauen zur Bewältigung der digitalen Komplexität mit ihren Auswirkungen auf das organisationale Arbeitsgefüge, als auch innovative Kompetenzen im Umgang mit den zur Verfügung stehenden Zeit- und Handlungsspielräumen (Hellert et al. 2018; Müller et al. 2017).

\subsection{Methode}

Im Rahmen der Erhebung ( $n=27)$ wurden zwölf leitfadengestützte Experteninterviews, zwei Gruppendiskussionen sowie eine Beobachtung durchgeführt (im Folgenden als Interviews zusammengefasst). Die untersuchten KMU arbeiten zu einem hohen Grad virtuell und stammen aus der IT-Branche. 
Die Auswertung erfolgte anhand der qualitativen Inhaltsanalyse nach Mayring. Nach der Transkription und Anonymisierung (Veränderung von Namen) erfolgte die Kodierung in Textsegmente, damit diese im Anschluss inhaltlich strukturiert und zusammengefasst werden konnten. Die Hauptkategorien „Zeitkompetenz“ und „Vertrauen" standen im Fokus der Analyse und wurden über einen mehrstufigen Prozess auf wenige Unterkategorien reduziert.

Im Ergebnisteil des Beitrags werden einzelne Subkategorien aus Zeitkompetenz und Vertrauen herausgegriffen, welche in Zusammenhang mit dem „Abgeben von Kontrolle“ stehen. Eine Subkategorie von Zeitkompetenz wurde als Überlast kodiert. Da mit Überlast in der Stichprobe eine der größten Herausforderungen im Umgang mit Zeit- und Handlungsspielraum identifiziert wurde, wurde diese Subkategorie eingehend analysiert. Gemäß dem zusammenfassenden Vorgehen erfolgte ein weiterer Schritt, in dem Aussagen systematisch gruppiert wurden.

Die abgeleiteten Handlungsempfehlungen basieren auf den Ergebnissen der qualitativen Studie und Unternehmensworkshops, bei denen Ergebnisse und Handreichungen hinsichtlich ihrer Praxistauglichkeit geprüft wurden.

\subsection{Ergebnisse}

In der qualitativen Inhaltsanalyse wurden einige Themenbereiche identifiziert, die sich voneinander abgrenzen lassen. Zeitkompetenz und Vertrauen sind gemäß den Befragten wichtig für virtuelle Teamarbeit und scheinen sich gegenseitig zu beeinflussen. In Bezug auf den Zeit- und Handlungsspielraum wurden verschiedene Aussagen getroffen, welche die hohe Relevanz von Vertrauen nahelegen. Eine Herausforderung ist besonders aufgefallen, da sich insbesondere Führungskräfte wiederholt damit konfrontiert sehen. Die Aussagen zu diesem Problemfeld werden in den folgenden Abschnitten gebündelt und durch Zitate aus den Gruppendiskussionen und Interviews verdeutlicht. Dabei ist zu berücksichtigen, dass die Analyse des Zeit- und Handlungsspielraums mit einem Fokus auf Zeit bzw. Umgang mit Zeit durchgeführt wurde. Hier ist der Aspekt selbstbestimmter Zeiteinteilung von besonderem Interesse.

\subsubsection{Vertrauen ermöglicht Zeit- und Handlungsspielraum}

Als Grundlage für eine funktionierende Teamarbeit wird von vielen Befragten eine eigenständige Arbeitsweise beschrieben, die von einem hohen Zeit- und Handlungsspielraum geprägt ist. Als Voraussetzung dafür wird Vertrauen im Team und durch Führungskräfte genannt.

Eine selbstbestimmte Arbeitsweise ist gemäß einer Teilnehmerin in einer Gruppendiskussion nur aufgrund des Vertrauens durch ihren Vorgesetzten möglich: „Selbst der Herr Willner fragt uns wenig, was wir tun. Anscheinend kommen wenig Beschwerden... Also 
er traut uns.“ (Gruppendiskussion 2, 19) Dieses von der Teilnehmerin angenommene Vertrauen wurde zudem durch folgende Aussage unterstrichen: „Wir haben das auch so abgesprochen, ich sag[e]: ,Herr Willner, wollen Sie jede Mail von mir sehen?` Der sagt: ,um Gottes Willen!‘. Also er vertraut da auch wieder.“ (Gruppendiskussion 2, 82) Später sagte die Führungskraft zu selbstorganisierter Arbeit, dass es in diesem Prozess wichtig ist, Verantwortung abzugeben: ,das funktioniert auch nur, wenn ich den Leuten vertraue und wenn ich es aushalte, dass es nicht ,mein Weg' ist, sondern ,ein Weg‘." (Gruppendiskussion 2, 505).

Die eigene Arbeit selbst zu strukturieren und die Zeitnutzung selbst zu bestimmen ist bei einem hohen Zeit- und Handlungsspielraum möglich. Dieser ist gemäß den Interviews sehr stark von dem Vertrauen durch Vorgesetzte abhängig. Zudem ist der Zeitund Handlungsspielraum für die Befragten notwendig, um die Aufgaben bewältigen zu können. „Wir müssen ja selber wissen, was wir zu tun haben.“ (Interview 06, 178) So bringt es ein Befragter auf den Punkt: „Wenn man sich einigermaßen gut organisieren kann, hat man bei uns im Unternehmen wirklich die Möglichkeiten sich das auch so einzuteilen, wie man es braucht. Also, mein Chef möchte von mir keine Abrechnung haben, [im Sinne von:], wann hast du genau was gemacht und wofür hast du wie viel Zeit investiert?““(Interview 01, 336).

Es lässt sich zusammenfassen, dass ein hoher Zeit- und Handlungsspielraum in den betrachteten Unternehmen als wichtig eingestuft wird. Führungskräfte greifen dabei wenig in die Arbeitsweise ein, solange die gesetzten Ziele erreicht werden. Hierbei stehen Führungskräfte vor der Herausforderung, ihr Vertrauen in Bezug auf die Zeitnutzung zu erteilen. Eine Einschätzung, wie viel Vertrauen jeweils angemessen ist, erfolgt auf Basis der vergangenen Erfahrungen. Dies wird in folgender Aussage verdeutlicht: ,ich denke schon, dass das [Vertrauen] schon da ist, aber nichtsdestotrotz wird es erst wachsen, wenn man länger miteinander arbeitet und sich näher kennenlernt. Ich glaube, das ist ansteckend.“ (Interview 02, 253).

\subsubsection{Verantwortungsvoller Umgang mit Zeit- und Handlungsspielraum: Problem Überlast}

In den betrachteten Unternehmen ist insgesamt ein hoher Zeit- und Handlungsspielraum zu beobachten. Gleichzeitig wird das Problem einer hohen Auslastung bzw. einer Überlast-Situation von einigen Befragten thematisiert. Aufgrund des variierenden Umgangs mit Zeit liegt nahe, dass eine zu hohe Auslastung bei zu wenigen zur Verfügung stehenden Ressourcen eine Gefahr für die Beschäftigten darstellt, die unterschiedlich erlebt wird. Bei Überlast liegt jedoch eine Schwierigkeit in der Feststellung von Gründen. Dabei stellen sich die Fragen, ob tatsächlich externe Gründe vorliegen und ob Personen offen damit umgehen. Die folgenden Absätze beschäftigen sich mit Interviewaussagen zu diesen Fragen. 


\subsubsection{Gründe für Überlast}

Auf Basis der zusammengefassten Interviewaussagen lassen sich einige Gründe für Überlast identifizieren. Dazu zählen Informationsflut, eine starke Ergebnisorientierung, Entgrenzung aufgrund hoher Verantwortung, Leistungsdruck sowie Personalengpässe. Durch diese Faktoren kann Zeitdruck entstehen, der bei anhaltender Belastung zu einer Überlast werden kann. Dieser Zustand zeigt sich an folgender Aussage: „'Ich traue mich gar nicht einen Termin zu machen, Sie sind ja immer zu.' Obwohl die dringend notwendig wären. Das sind so Sachen, so eine Herausforderung, vor der wir alle stehen." (Gruppendiskussion 2, 38) Beim Umgang mit den Belastungen gibt es differenzielle Unterschiede, wie an folgender Aussage deutlich wird: ,... ich glaube nicht daran, dass eine Person generell schlecht ist, die passt einfach nicht in die Situation hinein, also [das] Schlüssel-Schloss-Prinzip funktioniert nicht. Also nochmal den Schlüssel ändern oder das Schloss und das ist Arbeit, ganz konkrete - vor Ort." (Interview 10, 48) In einem weiteren Interview weist ein Befragter darauf hin, dass es die Aufgabe von Führungskräften ist, auf die Einhaltung der Arbeitszeit zu achten, sodass nicht zu viel Mehrarbeit geleistet wird. (Interview 11, 101).

Anhand der Aussagen wird deutlich, dass Gründe für Überlast in den Personen, in organisationalen Aspekten sowie in anderen externen Faktoren liegen können.

\subsubsection{Umgang mit Überlast}

Überlastsituationen werden in den Unternehmen meist erst spät erkannt und auch wenn sie bekannt sind, werden oft unzureichende Maßnahmen getroffen. Es scheint, als würde ein Gewöhnungseffekt eintreten, sodass ursprünglich als beanspruchend wahrgenommene Situationen zur Normalität werden. In Abgrenzung zu der als negativ beurteilten Überlastung berichten die Befragten, dass arbeitsintensive Phasen durch den Produktzyklus bedingt sein können. Diese Ausnahmesituationen stellen für die Beschäftigten kein Problem dar, wenn im Anschluss ein Freizeitausgleich stattfindet.

Eine hohe Identifikation mit der eigenen Arbeit bzw. intrinsische Motivation können dazu führen, dass eine Überschreitung der eigenen Belastbarkeitsgrenze erst spät bemerkt wird. So berichtet ein Interviewter: „... manche Leute empfinden ja so einen negativen Stress, das empfinde ich noch gar nicht mal, aber man merkt eben am Ende trotzdem, dass der Körper dann ....wahrscheinlich nicht mehr so will. Du kannst eben nicht auf Dauer dann immer so.“ (Interview 06, 91) Betroffene können individuell mit den hohen Anforderungen umgehen, indem sie ihre persönlichen Ressourcen im Umgang mit Stress steigern. Neben einer gelassenen Einstellung sind verschiedene Techniken im Umgang mit Zeit hilfreich. Dabei wurde eine strukturierte Arbeitsweise genannt, um unter Zeitdruck nicht den Überblick zu verlieren. Auch ein organisationales Gesundheitsmanagement mit regelmäßigen Angeboten kann bei der Bewältigung von Stress unterstützen.

Häufiger wurde jedoch genannt, dass die Anforderungen angepasst werden sollten, da diese das grundlegende Problem darstellen. Das heißt, dass sich Teammitglieder gegenseitig entlasten können oder Aufgaben umverteilt werden. Die Unternehmen und 
Führungskräfte tragen dabei eine große Verantwortung. Zudem sind Arbeit und Privatleben in der modernen Arbeitswelt verflochten, was bedeutet, dass sich eine Überforderung im Privatleben auf das Arbeitsleben auswirken kann und umgekehrt. Ein Interviewter erzählte davon, dass Mitarbeitende die verschiedenen Lebensbereiche integrieren: ,schon mal passiert, dass mir dann jemand hinterher in einem persönlichen Gespräch erzählt hat, dass es persönliche Gründe hatte, warum da mal so ein Durchhänger da war... dann nehme ich denjenigen auch mal aus der Schusslinie, bzw. dann ist es auch legitim, wenn jemand dann mal nicht $100 \%$ bringt.“ (Interview 11, 164) Teams oder Personen mit Entscheidungsbefugnis können bei starker Auslastung Einfluss darauf nehmen, um der Entstehung von Stress entgegen zu wirken: „Das heißt, jeder Kunde, der jetzt kommt und noch was haben will, der muss bis nächstes Jahr warten. Und damit haben die sich auch so ein bisschen den Druck genommen, jetzt noch was aufnehmen zu müssen. Die Erwartungshaltung bei den Adressaten wird quasi gesenkt.“ (Interview 11, 65) Auf organisationaler Ebene kann ein Ansatz darin liegen, Arbeitslast zwischen Teams und Mitarbeitenden umzuverteilen, sodass die Auslastung möglichst homogen ist. (Interview 1, 318).

\subsubsection{Auswirkungen von Überlast}

Die Beobachtung zeigt ein einheitliches Bild im Unternehmensalltag: Terminkalender sind voll, es gibt kaum Tage, die nicht bereits gefüllt sind. Neue Aufgaben sollen nach Meinung Einzelner sofort erledigt werden. Die Beschäftigten können leicht den Überblick verlieren und Persönliches bleibt oftmals auf der Strecke. In Tab. 7.1 ist eine Übersicht über verschiedene Auswirkungen von Überlast mit passenden Interviewaussagen dargestellt.

Die Überforderung durch anhaltenden Zeitdruck ist der Grund, dass manche Aufgaben zurückgestellt werden und dringliche Tätigkeiten Vorrang haben. Die Befragten, die von Überlast betroffen waren, berichten von einhergehender Erschöpfung und Krankheit bzw. Präsentismus. Diese Schilderungen betreffen das individuelle Erleben. Darüber hinaus wirken sich Überlastungssituationen auf die Team-Ebene aus. Der Zeitdruck führt dazu, dass weniger Kommunikation stattfindet, längere Wartezeiten auftreten und nicht alle Aufgaben gründlich bearbeitet werden können. Auf organisationaler Ebene sind Auswirkungen in Form von deutlich erhöhten Fehlzeiten und einer hohen Fluktuation beobachtbar.

\subsubsection{Wahrnehmung von Überlast}

Aus organisatorischer Perspektive wurde in Bezug auf Überlast gesagt: „Jeder Mensch hat auch einen anderen Level wo für ihn der Stress beginnt, wenn eben, das kann man glaube ich nicht verallgemeinern, wichtig ist, dass man die Mitarbeiter sensibilisiert und auch die Vorgesetzten sensibilisiert, dass man es einfach merkt, wenn Hinweise kommen, ob jetzt so ein Kurs im Zeitmanagement oder Coaching immer tatsächlich auch das gewünschte Ergebnis bringt, ist sehr, sehr schwierig." (Interview 07, 169) Um dies aufzugreifen, können Führungskräfte mit den Teammitgliedern Kontakt halten und 
Tab. 7.1 Zusammenfassung der genannten Auswirkungen von Überlast

\begin{tabular}{|c|c|}
\hline Auswirkung & Zitat \\
\hline Nur die nötigsten Aufgaben erledigt & $\begin{array}{l}\text { „Das waren dann wirklich nur operative } \\
\text { Sachen, also man konnte sich dann auch gar } \\
\text { nicht um konzeptionelle Sachen kümmern.““ } \\
\text { (Interview 12, 202) }\end{array}$ \\
\hline Überforderung & $\begin{array}{l}\text { „Ich habe mich im Januar dann auch mal eine } \\
\text { Woche rausnehmen lassen, weil ich wirklich } \\
\text { nicht mehr wusste, noch ein, noch aus, nicht } \\
\text { mehr richtig geschlafen und so etwas und habe } \\
\text { dann halt für mich jetzt auch Konsequenzen dann } \\
\text { gezogen, dass ich halt sage, dass ich die Themen } \\
\text { mehr priorisiere“ (Interview 12, 200) }\end{array}$ \\
\hline Erschöpfung & $\begin{array}{l}\text { „Ich habe das nie selbst als so negativ } \\
\text { empfunden und trotzdem merkt man, dass der } \\
\text { Körper dann doch nicht mehr so will und wo } \\
\text { ich dann auch Angst hatte...“ (Interview 06, } \\
\text { 96) }\end{array}$ \\
\hline Präsentismus & $\begin{array}{l}\text { „Ich habe mich total schlecht gefühlt und ich war } \\
\text { da auch noch krank eigentlich und [ich] habe da } \\
\text { ganz viele Tabletten dann auch noch genommen. } \\
\text { Weil ich von einem Tag zum anderen Tag [das } \\
\text { Thema] beenden musste und die mir zu kurz- } \\
\text { fristig Bescheid gegeben hatten und das muss } \\
\text { immer ... in dem Fall abgestimmt werden. Und } \\
\text { die Fälle waren aber an sich so gravierend, dass } \\
\text { wir das wirklich machen mussten..." (Interview } \\
\text { 06, 118) }\end{array}$ \\
\hline $\begin{array}{l}\text { Zeit für Kommunikation, Meetings, Vor- } \\
\text { bereitung von Meetings, Bearbeitung von } \\
\text { E-Mails, Persönliches, Pausen, strategische } \\
\text { Themen und Teamlernen fehlt }\end{array}$ & $\begin{array}{l}\text { „Jeder Mitarbeiter darf selber Punkte } \\
\text { ins Protokoll anmelden. Das ist in allen } \\
\text { Themen so. Ich will das besprechen. Und die } \\
\text { Schwierigkeit ist in dem System, dass wir } \\
\text { unterschiedliche Aggregatszustände haben, } \\
\text { also erstens mal so strategische Grund- } \\
\text { satzdinge und dann auf einmal [operative } \\
\text { Fragen]..., ich schaffe das nie im Vorfeld, das } \\
\text { ordentlich zu filtern, ... Ich schaffe das einfach } \\
\text { nicht.“ }(\mathbf{1 0 , 5 8 )}\end{array}$ \\
\hline
\end{tabular}

sollen ihre Empathie einsetzen, um Überlastsituationen auch bei virtueller Arbeit wahrzunehmen. Diese sind laut den Befragten bereits an kleinen Verhaltensänderungen oder Indizien bemerkbar. Beispielsweise können der Zeitstempel einer E-Mail in den Abendstunden oder eine hohe Termindichte im Kalender ein Hinweis sein. Auch ein kurzer Anruf kann bei der Einschätzung bereits helfen: „Ob sie damit zurechtkommt, ja, mit dem Arbeitspensum und darüber kommt man ins Gespräch und zugleich stelle ich auch 
fest, dass Kollegen feinfühlig sind, wenn es andersherum mich mal betrifft, dass Nachfragen an mich kommen, wie es mir damit geht.“ (Interview 05, 157).

Führungskräfte sind gefragt, im Umgang mit Zeit anzuleiten, wenn sich die Mitarbeitenden selbst zu sehr verausgaben. Dazu erklärt eine Führungskraft, wie sie mit dieser Herausforderung umgeht: „Also da muss man immer wieder mit der Person sprechen und sagen: ,Du darfst dich nicht rausreißen lassen, die Dinge müssen am besten über mich laufen, ich kann besser einschätzen, wer in der Abteilung noch ein bisschen mehr Puffer hat, um mit dem anderen zu sprechen. ‘... Also, wenn das Arbeitspensum dann nicht so voll ist, dann fällt das nicht so auf. Und sobald es dann wieder alles voll ist mit Terminen, da muss man wieder einschreiten." (Interview 02, 209).

Folgende Kriterien, an denen Überlast frühzeitig erkannt werden kann, wurden von den Befragten genannt:

- Verändertes Verhalten bzw. soziale Interaktion ist verändert, z. B. Ton bei Telefonaten ist verändert oder Konzentration ist gestört, Gereiztheit oder „kurz angebunden sein“ in Telefonaten

- Mehr Arbeit wird an Kollegen/innen abgegeben

- Weniger dringliche Aufgaben werden zurückgestellt

- Wenig bis gar keine Zeit für Vorbereitung von Meetings oder Workshops

- Unzufriedenheit mit erzielten Ergebnissen

- Pause wird verspätet oder gar nicht angetreten

- Erhöhte Fluktuation und Fehlzeiten

\subsubsection{Umgang mit Arbeitszeit: Typisierung der Mitarbeitenden nach Umgang mit Überlast}

Fazit der Auswertung ist, dass Überlast ein zentrales Problem im Kontext eines hohen Zeit- und Handlungsspielraums darstellt. Es ist schwer zu erkennen, ob Personen tatsächlich betroffen sind. Da es von den Personen individuell abhängt, muss hier differenziert werden. Eine Typisierung der Mitarbeitenden nach dem Umgang mit Zeit kann Entscheidungsträgern helfen, auf die individuellen Bedürfnisse einzugehen.

Die Typisierung kann nach zwei Kriterien vorgenommen werden (vgl. Tab. 7.2). Zum einen beeinflusst die Einstellung der Betroffenen den Umgang: hier stellt sich also die

Tab. 7.2 Vier-Felder-Tafel zur Typisierung im Umgang mit Überlast

\begin{tabular}{l|l}
\hline Überlast ,ja“, Thematisieren ,ja“ „Problem solver“ & $\begin{array}{l}\text { Überlast ,ja“, Thematisieren „,nein“ } \\
\text { „Problem denier“6 }\end{array}$ \\
\hline Überlast „nein“, Thematisieren ,ja“ „Problem seeker“ & $\begin{array}{l}\text { Überlast „nein“, Thematisieren } \\
\text { „nein“ } \\
\text { „Healthy workers“ }\end{array}$ \\
\hline
\end{tabular}


Frage, ob sie Überlast thematisieren; zum anderen ist zunächst festzustellen, ob objektiv eine Überlastsituation vorliegt. Diese Typisierung ist als Modell zu verstehen, welches Anhaltspunkte für eine Differenzierung gibt - die Grenzen sind dabei fließend.

\subsection{Empfehlungen für die Gestaltung virtueller Führung}

Aus den Ergebnissen geht hervor, dass Überlast ein zentrales Problem bei vielen virtuell Arbeitenden darstellt. Die Rolle von Führungskräften verändert sich und ihr Eingreifen in neuen Situationen ist gefragt. Aufgrund der Interviews und Gruppendiskussionen ist klar zu erkennen, dass das erforderliche Umdenken in Bezug auf Zeit- und Handlungsspielraum Offenheit und Sensibilität erfordert.

Welches Mindset für virtuelle Führung angemessen ist, lässt sich anhand des qualitativen Vorgehens beantworten: Führungskräfte sollten proaktiv Kontakt mit den Mitarbeitenden halten, an deren Wohlbefinden interessiert sein und ein Gespür dafür entwickeln, in welchen Aspekten sie den Teammitgliedern vertrauen können. In diesen Aspekten kann und soll Verantwortung bzw. Kontrolle abgegeben werden. Um möglichst gut mit verschiedenen Situationen umgehen zu können, bieten sich einige Strategien an, die in den folgenden Abschnitten dargestellt sind.

\subsubsection{Gestaltungsansätze für genannte Herausforderungen}

Eine Herausforderung besteht in der Förderung von Vertrauen und der Kommunikation in virtuellen Teams. Aus den Interviews geht hervor, dass diese Herausforderung bewältigbar ist und sogar das gleiche Vertrauensniveau erreicht werden kann wie in Präsenzteams. Die Bedingungen dafür sind zwei wesentliche Punkte: Zeit und Raum. Um ein hohes Vertrauensniveau in virtuellen Teams zu erreichen, wird Zeit benötigt. Soll das Team also nur für ein paar Wochen oder Monate zusammenarbeiten, dann kann ein Vertrauensvorschuss sinnvoll sein. Jedoch war diese kurzzeitige Projektarbeit in der betrachteten Stichprobe nicht vertreten. Analog zu Teamentwicklungsprozessen (Tuckman und Jensen 2010) ist davon auszugehen, dass sich die Entwicklung in virtuellen Teams langsamer abspielt als in Präsenzteams. Der zweite wesentliche Ansatz liegt im Raum: wenn Teammitglieder die Möglichkeit haben, sich persönlich kennenzulernen, gibt es i. d. R. einen Sprung in der Vertrauensentwicklung. Reisezeit und -kosten sind demnach als Investition in die Teamentwicklung zu betrachten. Bei einem hohen Vertrauensniveau ist davon auszugehen, dass sich auch die Kommunikation positiv entwickelt.

Wenn virtuell arbeitende Teams in ihrer Teamentwicklung noch nicht weit fortgeschritten sind, jedoch das entsprechende Mindset vertreten, dann können die Teammitglieder durch verschiedene Maßnahmen aktiv an der Entwicklung von Zeitkompetenz 
und Vertrauen arbeiten. Dazu sei auf den Kompass - Zeit \& Vertrauen ${ }^{1}$ verwiesen, in dem sich viele unterschiedliche Anregungen für verschiedene Situationen finden. Er wurde in Zusammenarbeit mit Praxispartnern entwickelt und von den Unternehmen im Test als hilfreich im Aufbau von Zeitkompetenz und Vertrauen bewertet. Wichtige Forschungsergebnisse wurden dabei aufgegriffen: Förderung wertschätzender Kommunikation, Führungskompetenz in virtuellen Arbeitsstrukturen, Erreichbarkeit, verbindliche Regelungen sowie Impulse zur Förderung von Kohäsion.

Im Rahmen des beschriebenen Mindsets gibt es einige konkrete Handlungen, die aktiv verfolgt werden können. Diese können Führungskräften im Rahmen von Trainings oder sensibilisierenden Gesprächen vermittelt werden. Eine Übersicht über zentrale Gestaltungsansätze findet sich in Tab. 7.3.

\subsubsection{Führung bei unterschiedlichem Umgang mit Überlast}

Die Zeit, die Führungskräfte nutzen, um anzuleiten, richtet sich nach dem Bedarf bei den einzelnen Personen. Beispielsweise brauchen Beschäftigte, die neu im Unternehmen anfangen meistens mehr Aufmerksamkeit als Mitarbeitende, die dort schon seit Jahren beschäftigt sind. Der Befürchtung, das Vertrauen könne ausgenutzt werden oder Mitarbeitende könnten sich bei hoher Eigenverantwortlichkeit selbst überfordern, kann anhand einer möglichst objektiven Einschätzung entgegengewirkt werden. Dazu dient die Vier-Felder-Tafel (Tab. 7.2).

Im letzten Beispiel in Tab. 7.3 zeigt sich eine Situation, die nicht leicht zu bewältigen ist: Eine Führungskraft stellt fest, dass ein Teammitglied überlastet ist und Unterstützung benötigt. Wie bereits aufgeführt, kann unterteilt werden, ob Überlast vorliegt und ob Betroffene offen damit umgehen. Damit ein situativer Umgang möglich ist, empfehlen sich unterschiedliche Herangehensweisen für die vier Kombinationen. Die Typisierung ist verallgemeinert und gibt eine Tendenz wieder. Bei der Anwendung sollte eine Adaption entsprechend der individuellen Unterschiede der betroffenen Person erfolgen.

\subsubsection{Führung von Problem Solvers}

Mitarbeitende, die überlastet sind und dies offen thematisieren, die Problem Solvers, wollen etwas an ihrer Situation ändern. Führungskräfte können ihnen dabei helfen, indem sie die Anliegen ernst nehmen und bei der Umsetzung organisational unterstützen. Sie können anregen, Ressourcen und Anforderungen zu analysieren und Fehlbelastungen zu reduzieren. Führungskräfte können zudem als Vorbild fungieren, indem sie Zeitkompetenz und Gelassenheit vorleben.

\footnotetext{
${ }^{1}$ Der Kompass - Zeit \& Vertrauen ist im Rahmen des Projekts vLead entstanden und kann unter www.vLead.de als PDF heruntergeladen werden.
} 
Tab. 7.3 Handreichung für die Gestaltung virtueller Teamarbeit

\begin{tabular}{|c|c|c|}
\hline Ziel & Handlung & Beispiel \\
\hline $\begin{array}{l}\text { Führen } \\
\text { offener } \\
\text { Gespräche }\end{array}$ & $\begin{array}{l}\text { Legen Sie ein regelmäßiges } \\
\text { Intervall fest, in dem Sie } \\
\text { Teammitglieder anrufen. } \\
\text { Dabei können Sie Inhalte } \\
\text { thematisieren, die über den } \\
\text { Arbeitsalltag hinausgehen. } \\
\text { Anhand solcher offenen } \\
\text { Gespräche wird es Ihnen } \\
\text { leichter fallen, empathisch auf } \\
\text { die Teammitglieder einzugehen }\end{array}$ & $\begin{array}{l}\text { Eine Führungskraft ruft ein } \\
\text { Mal pro Monat ein Team- } \\
\text { mitglied an und beide sprechen } \\
\text { über Persönliches, wie z. B. } \\
\text { die Gestaltung des Wochen- } \\
\text { endes. Im nächsten Monat } \\
\text { wird ein anderes Teammitglied } \\
\text { angerufen }\end{array}$ \\
\hline $\begin{array}{l}\text { Gestaltung von } \\
\text { Kommunikationsstrukturen }\end{array}$ & $\begin{array}{l}\text { Halten Sie ein Meeting (in } \\
\text { Präsenz oder als Video- } \\
\text { konferenz) ab, in dem es nur } \\
\text { um die Kommunikation im } \\
\text { Team geht. Dabei können ver- } \\
\text { schiedene Aspekte, wie etwa } \\
\text { die Erreichbarkeit oder die } \\
\text { Verbindlichkeit von Zusagen, } \\
\text { thematisiert werden }\end{array}$ & $\begin{array}{l}\text { Ein Team trifft sich ein Mal } \\
\text { pro Jahr an einem zentral } \\
\text { gelegenen Ort. Der Nach- } \\
\text { mittag wird für die Reflexion } \\
\text { der Kommunikation im Team } \\
\text { genutzt. Dabei wird } \\
\text { z. B. angesprochen, dass oft } \\
\text { unklar ist, wer für bestimmte } \\
\text { Aufgaben zuständig ist }\end{array}$ \\
\hline $\begin{array}{l}\text { Entwicklung von } \\
\text { Transparenz }\end{array}$ & $\begin{array}{l}\text { Machen Sie alle Informationen } \\
\text { dem gesamten Team zugäng- } \\
\text { lich. Das zeigt zum einen Ver- } \\
\text { trauen, zum anderen können } \\
\text { Teammitglieder so voneinander } \\
\text { lernen }\end{array}$ & $\begin{array}{l}\text { In der Cloud sind } \\
\text { alle Dokumente und } \\
\text { Präsentationen thematisch } \\
\text { sortiert abgelegt. Wenn eine } \\
\text { Person z. B. ein Angebot } \\
\text { einholen möchte, kann sie auf } \\
\text { die Formulierung in älteren } \\
\text { Angeboten zurückgreifen }\end{array}$ \\
\hline $\begin{array}{l}\text { Entwicklung von E-Leader- } \\
\text { ship-Kompetenz }\end{array}$ & $\begin{array}{l}\text { Setzen Sie sich aktiv damit } \\
\text { auseinander, in welchen } \\
\text { Handlungsfeldern Sie sich } \\
\text { weiterentwickeln können: } \\
\text { in der Beziehung zu Team- } \\
\text { mitgliedern, im Punkt Zeit- } \\
\text { und Handlungsspielraum, in } \\
\text { Bezug auf Koordination und } \\
\text { Orientierung oder bei der } \\
\text { individuellen Entwicklung der } \\
\text { Teammitglieder }\end{array}$ & $\begin{array}{l}\text { Eine Führungskraft ist sich } \\
\text { nicht sicher, ob den Team- } \\
\text { mitgliedern zu viel Zeit- und } \\
\text { Handlungsspielraum gewährt } \\
\text { wurde, weil sie ,gestresst“ } \\
\text { wirken. In Gesprächen ver- } \\
\text { sucht die Führungskraft, die } \\
\text { Einschätzung zu überprüfen. } \\
\text { Bei einem Teammitglied zeigt } \\
\text { sich tatsächlich eine Überlast } \\
\text { und Schritte zur Entlastung } \\
\text { werden eingeleitet }\end{array}$ \\
\hline
\end{tabular}




\subsubsection{Führung von Problem Deniers}

Diese Maßnahmen umzusetzen ist für Problem Deniers schwieriger. Sie sind zwar auch von Überlast betroffen, wollen aber i. d. R. nicht darüber sprechen oder sehen selbst kein Problem. Dies kann u. a. an einem übersteigerten Leistungsmotiv oder einer starken Karriereorientierung liegen. Wenn Führungskräfte oder Teammitglieder bemerken, dass Mitarbeitende über einen längeren Zeitraum zu viel arbeiten, dann können sie ihre Beobachtung der betroffenen Person mitteilen. In einem offenen und wertschätzenden Gespräch können mögliche Lösungen thematisiert werden.

\subsubsection{Führung von Problem Seekers}

Einen Sonderfall stellen Personen dar, die sagen, dass sie unter Überlast leiden, obwohl dies objektiv nicht nachvollziehbar ist. Die Problem Seekers sind entweder tatsächlich davon überzeugt überlastet zu sein oder sie geben es vor. Für Führungskräfte ist es dabei wichtig herauszufinden, welches Motiv sich hinter der Einstellung verbirgt. Gespräche mit den Betroffenen oder Teammitgliedern können bei einer Einschätzung helfen. Eventuell braucht die oder der Problem Seeker eine Schulung zur Förderung von Zeitkompetenz oder Stressbewältigung. Um jedoch lösungsorientiert vorgehen zu können, ist sowohl aufseiten der Führungskräfte als auch der Mitarbeitenden ein passendes Mindset notwendig.

\subsubsection{Führung von Healthy Workers}

Personen, die nicht überlastet sind und einen offenen Umgang pflegen, brauchen grundsätzlich weniger Aufmerksamkeit. Ihr Status kann sich jedoch verändern, daher ist zu empfehlen, sie im Blick zu behalten und ihnen Anerkennung für ihr Engagement zu zeigen. Maßnahmen sind nicht erforderlich.

Führung auf Distanz unterliegt anderen Anforderungen als in Präsenzteams. Daher gilt es, diese Anforderungen als gegeben zu akzeptieren und entsprechende Ressourcen zur Bewältigung zur Verfügung zu stellen. Schließlich hat virtuelle Teamarbeit viele Vorteile. Die Mitglieder virtueller Teams können mitunter ihren Wohnort frei wählen und bei Home-Office Fahrzeiten reduzieren. Unternehmen können Experten aus unterschiedlichen Standorten leichter zusammenbringen. Flexible Arbeitszeitmodelle sind möglich, wenn Teams aufgrund des zeitlichen Aspekts virtuell arbeiten. Manche berichten sogar, dass sie sich bei virtueller Teamarbeit besser konzentrieren können.

Virtuelle Teamarbeit birgt zwar Risiken, jedoch können sie reduziert werden, wenn die obigen Empfehlungen berücksichtigt werden. Beispielsweise liegt es in der Verantwortung von Führungskräften, auf die Gesundheit und Auslastung der Mitarbeitenden zu achten. Ein Umgang mit den neuen Anforderungen ist erlernbar, wie Berichte aus den untersuchten Unternehmen zeigen. Es lohnt sich also, die Vorteile von virtueller Teamarbeit zu nutzen, da diese Arbeitsweise sich auszahlt, wenn sie einmal erlernt und umgesetzt wurde. Außerdem zeigten die Interviews analog zur Feldtheorie, dass sowohl Person als auch Situation bzw. Umgebung das Verhalten bestimmen (Lewin 2013). Dies zeigt sich auch bei Führung auf Distanz. 
Die Empfehlungen gelten v. a. für Führungskräfte, jedoch hat sich in der Unternehmenspraxis gezeigt, dass auch andere Teammitglieder Verantwortung übernehmen können. In Unternehmen im Projekt vLead war während eines Zeitraums von zwei Jahren ein Wandel hin zu selbstorganisierten Teams zu beobachten. Dabei wurde klar: Die Rolle von Führungskräften besteht zunehmend darin, als ,servant leader“ ein selbstorganisiertes Team wertschätzend und respektvoll zuunterstützen (Pörksen und Schulz von Thun 2020, S. 37). Dies reicht so weit, dass der Begriff „Führung“ innerhalb der Teams infrage gestellt wird, obwohl Personen explizit Führungsaufgaben übernehmen. Große Zeit- und Handlungsspielräume können folglich in der Praxis zu gut funktionierender Teamarbeit beitragen. An dem beobachteten Wandel wird auch das Mindset für virtuelle Teamarbeit deutlich, welches von der Bereitschaft zum Perspektivund Paradigmenwechsel und stetiger Kompetenzentwicklung sowie flachen Hierarchien geprägt ist.

\section{Literatur}

Ale Ebrahim N, Ahmed S, Taha Z (2009) Virtual teams: a literature review. Aust J Basic Appl Sci 3(3):2653-2669

Avolio BJ, Kahai S, Dodge GE (2000) E-leadership. Implicationsfor theory, research, and practice. Leadersh Quart 11(4):615-668

Bakker AB, Demerouti E (2014) Job demands-ressources theory. In: Chen PS, Cooper LC (Hrsg) Work and wellbeing. Wellbeing: a complete reference guide III, Wiley-Blackwell, Chichester

Bennett EE (2010) The coming paradigm shift: synthesis and future directions for virtual HRD. Adv Developing Hum Resour 12(6):728-741. https://doi.org/10.1177/1523422310394796

Chudoba KM, Wynn E, Lu M, Watson-Manheim MB (2005) How virtual are we? Measuring virtuality and understanding its impact in a global organization. Inf Syst J 15(4):279-306

Cleary M, Hungerford C, Lopez V, Cutcliffe JR (2015) Towards effective management in psychiatric-mental health nursing: the dangers and consequences of micromanagement. Issues Mental Health Nurs 36(6):424-429. https://doi.org/10.3109/01612840.2014.968694

Germain ML, McGuire D (2014) The role of swift trust in virtual teams and implications for human resource development. Adv Dev Hum Resour 16(3):356-370

Grawe K. (2004) Psychological Therapy. Hogrefe \& Huber Publishers, Göttingen, Washington

Hellert U (2018) Arbeitszeitmodelle der Zukunft. Arbeitszeiten flexibel und attraktiv gestalten, 2. Aufl. Haufe, Freiburg

Hellert U, Krol B, Tegtmeier P (2013) Innovative Arbeitszeitgestaltung und Zeitkompetenz bei einem Studium neben dem Beruf. In: Hellert U (Hrsg) iap Schriftreihe, Bd 5. MA Akademie, Essen

Hellert U, Müller F, Mander R (2018) Zeitkompetenz, Vertrauen und Prozessfeedback im Virtual Work Resource Model. In: Hermeier B, Heupel T, Fichtner-Rosada S (Hrsg) Arbeitswelten der Zukunft. Springer, Wiesbaden

Hellert U, Sichert-Hellert W, Sträde K (2009) Präventive Arbeitsgestaltung zur Förderung der Beschäftigungsfähigkeit in der IT-Wirtschaft im Kontext von Arbeitszeit, Gesundheit und Stress. In: Gesellschaft für Arbeitswissenschaft e. V. (Hrsg) 55. Frühjahrskongress der Gesellschaft für Arbeitswissenschaft: Arbeit, Beschäftigungsfähigkeit und Produktivität im 21. Jahrhundert. GfA Press, Dortmund 
Hofert S (2018) Das agile Mindset Mitarbeiter entwickeln Zukunft der Arbeit gestalten. Springer, Wiesbaden.

Hollmann S, Hellert U, Schmidt KH (2005) Anforderungen an eine zielbezogene Selbststeuerung im Rahmen hochflexibler Arbeitszeitmodelle. Themenheft „Faktor Zeit“. Wirtschaftspsychologie 7(3):2005 (Mieg, Harald A. (Hrsg))

Karasek Jr RA (1979) Job demands, job decision latitude, and mental strain: Implications for job redesign. Administrative science quarterly: 285-308.

Lewin K (2013) Principles of Topological Psychology, Read Books Ltd

Lilian SC (2014) Virtual teams: opportunities and challenges for e-leaders. Procedia-Soc Behav Sci 110:1251-1261

Luhmann N (2009) Vertrauen, 5. Aufl. UVK, Konstanz, München

Müller F, Mander R, Hellert U (2017) Virtuelle Arbeitsstrukturen durch Vertrauen, Zeitkompetenz und Prozessfeedback fördern. Gruppe. Interaktion. Organisation. Z Angewandte Organisationspsychologie (GIO) 48(4):279-287

Pörksen B, Schulz von Thun F (2020) Die Kunst des Miteinander-Redens. Hanser, München

Schmidt K-H, Neubach B (2009) Selbstkontrollanforderungen als spezifische Belastungsquelle bei der Arbeit. Z Personalpsychologie 8:169-179

Tegtmeier P, Hellert U (2015) Wie gelingt die Erholung bei einem Studium neben dem Beruf? Z Arb Wiss 69(2015):1

Tuckman BW, Jensen MAC (2010) Stages of small-group development revisited. Group Facilitation: Res Appl J 10:43-48

Open Access Dieses Kapitel wird unter der Creative Commons Namensnennung 4.0 International Lizenz (http://creativecommons.org/licenses/by/4.0/deed.de) veröffentlicht, welche die Nutzung, Vervielfältigung, Bearbeitung, Verbreitung und Wiedergabe in jeglichem Medium und Format erlaubt, sofern Sie den/die ursprünglichen Autor(en) und die Quelle ordnungsgemäß nennen, einen Link zur Creative Commons Lizenz beifügen und angeben, ob Änderungen vorgenommen wurden.

Die in diesem Kapitel enthaltenen Bilder und sonstiges Drittmaterial unterliegen ebenfalls der genannten Creative Commons Lizenz, sofern sich aus der Abbildungslegende nichts anderes ergibt. Sofern das betreffende Material nicht unter der genannten Creative Commons Lizenz steht und die betreffende Handlung nicht nach gesetzlichen Vorschriften erlaubt ist, ist für die oben aufgeführten Weiterverwendungen des Materials die Einwilligung des jeweiligen Rechteinhabers einzuholen.

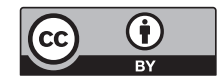

